\title{
Automatic Crack Detection of Road Pavement Based on Aerial UAV Imagery
}

\author{
Farzaneh Dadrasjavan ${ }^{1, *}$, Nima Zarrinpanjeh ${ }^{2}$ and Azam Ameri ${ }^{1}$ \\ 1 School of Surveying and Geospatial Engineering, University College of Engineering, University of Tehran, \\ Iran; \{fdadrasjavan,a.ameri\}@ut.ac.ir \\ 2 Department of Geomatics Engineering, Qazvin Branch, Islamic Azad University, Qazvin, Iran; \\ nzarrin@qiau.ac.ir \\ * Correspondence: fdadrasjavan@ut.ac.ir;
}

\begin{abstract}
Road surface monitoring more specifically crack detection on the surface of the road pavement is a complicated task which is found vital due to critical nature of roads as elements of transportation infrastructure. Cracks on the road pavement is detectable using remotely sensed imagery or car mounted platforms. UAV's are also considered as useful tools for acquiring reliable information about the pavement of the road. In This paper, an automatic method for crack detection on the road pavement is proposed using acquired videos from UAV platform. Selecting key frames and generating Ortho-image, violating non road regions in the scene are removed. Then through an edge based approach hypothesis crack elements are extracted. Afterwards, through SVM based classification true cracks are detected. Developing the proposed method, the generated results show $75 \%$ accuracy in crack detection while less than $10 \%$ of cracks are omitted.
\end{abstract}

Keywords: Crack Detection; UAV Imagery; SMV Classification; Aerial Photogrammetry

\section{Introduction}

Monitoring of Infrastructures and lifelines is believed to be a never ending story. As a matter of fact, the prominence of those facilities totally rely on perpetual and constant observance and evaluation of functionality of their elements. An undetected failure in water supplement in a populated region or any damage to main highways directly and immediately affects the life of residence. Therefore, a well- defined economic and efficient monitoring method for each and every important infrastructure and lifeline is believed to be vital.

Roads network are of the most prominent infrastructure that are systematically classified under the category of transportation lifelines. As Roads are the main transportation mean around the world, carrying goods and services is mostly based on developed and functional system of roads network. Roads are required to be functional almost every time. This eventually imposes tight minoring of the facility. On the other hand, due to the roads' distributed and elongated nature, the process of monitoring seems to be time consuming and expensive procedures. According to American Association of State Highway and Transportation Officials, maintenance of road cost more than $67 \mathrm{~B}$ US dollars annually (koch et al., 2015).

Roads are also vulnerable due to natural causes and disasters. Regardless of the more complex structures such as bridges and tunnels, the pavement of roads are also affected by natural and man caused events. Cracks are of the common damages to road pavement occurring mostly due to the constant change in temperature, weight of vehicles, erosion and chemical corruptions (Behnia et al., 2018). Consequently, crack monitoring seems to be promising to analyze and evaluate the current condition of roads. Quality, shape, type, depth and length of cracks are some of the most measures 
in crack analysis and monitoring (Mohan and Poobal, 2018). Therefore, acquiring reliable information about cracks on the surface of the roads seems to be vital for efficient maintenance of the road network.

The simplest method to inspect road surface is the visual inspection at the location which is considered to be inefficient and expensive. On the other hand, remote sensing techniques are believed to be one of the most suitable and reliable solutions for monitoring and maintenance of such facilities which are found interesting in nineties. With the advent of technology and the appearance of new imaging sensors, the concept of image based monitoring of roads has peeled and evolved. One of the most brilliant tools are Unmanned Airborne Vehicles also referred to as UAVs. Regardless of the diverse applications of UAVs, they are practically bolded in the process of acquiring high resolution imageries which facilitates more detailed inspection and monitoring through a safe and economic procedure (Aldea et al., 2015; Grandsaert, 2015; Sankarasrinivasan et al., 2015).

In this paper reviewing the most recent and the most important researches about crack detection from different sensors, a method to detect and extract asphalt cracks of roads using UAV based imagery is defined.

\section{Crack Detection from Airborne Imagery}

Automatic crack detection from imagery is categorized with respect to the data acquisition perspective and processing method (Coenen and Golroo, 2017). Considering data acquisition platform, three main imaging methods of terrestrial, Aerial and satellite images are used for remote sensing based crack detection. In terrestrial case, image acquisition systems are usually mounted on a moving vehicle such as cars and trains usually categorized under mobile mapping terms (Quintana et al., 2015). Airborne and satellite imaging systems are other information providing sources for crack detection (Schnebele et al., 2015). Due to the limitations of satellite imagery to provide sufficient spatial resolution, airborne systems and recently UAV based imaging systems are widely used for crack detection.

Crack detection techniques from images are mostly performed in four stages of preprocessing, segmentation, classification and enhancement. At the preprocessing level, basic enhancement techniques such as noise reduction, smoothing, sharpening and edge detection along with some more complex processing including removal of misleading and violating objects such as cars, vegetation, shades, signs and marks are performed. In the segmentation step, candidate crack primitives are extracted mostly based on the similarity of cracks to edge elements. In the next step classification is performed to define crack region and finally the results are enhanced mostly through morphological filtering (Cubero-Fernandez, 2017; Gavilan et al., 2011;).

Yokoyama et al., 2017 presented an automatics crack detection technique training artificial neural networks. The results showed in case of classification in two classes of crack and non-crack, the algorithm success rate in $79.9 \%$. In case of three classes of cracks, non - cracks and white lines, this method is 73.3 percent successful. It is also concluded that the method is successful in case of monitoring the stainless paved surfaces but inspecting concrete surfaces the successfulness of the method drops drastically.

Kim et al., 2017 proposed a crack detection method based on integrating different image analysis methods using both imagery and ultra-sonic sensors to measure distance. They focused on discrete crack and the main objective of paper was to measure length and width of cracks. The proposed system showed the successful detection of cracks thicker than $0.1 \mathrm{~mm}$ with the maximum length estimation error of $7.3 \%$.

Cubero-Fernandez et al., 2017 presents a crack detection technique using edge detection and morphological operations. In this research after preprocessing of spatial data through noise reduction and smoothing, canny edge detection is applied. The results are enhanced through morphological closing for gap removal. Finally, through a decision tree the success rate of $88 \%$ in achieved.

Ersoz et al., 2017 proposed a method extracting crack in two steps of segmentation and classification using images of a low traffic road segment. Thresholding the results, the remaining 
noise is removed via closing operator. SVM classifier issued to make distinguishes between cracks and non-cracks items considering geometric features. The accuracy of $97 \%$ was achieved where the flight was too low inspecting concrete surfaces.

Yin et al., 2015 proposed a crack detection technique by creating image pyramid of the acquired UAV images and performing canny edge detection in multiple scales. Final edges representing cracks are detected by aggregating the results of crack detection in different scales.

In Aldea and Le Hégarat-Mascle, 2015 crack detection is studied based on two algorithms of minimum path cost analysis and image percolation. Moreover, an improved strategy based on acontrario modeling which able to withstand the significant motion blur is proposed. Experimenting the proposed method on real image databases, the results showed that the proposed method is successful compared to other similar solution which only work on perfect images and not of significantly degraded images.

Chamben et al., 2010 considers a simulated imagery for crack detection. In this approach Markov model and adaptive filtering are used for segmentation and crack detection. In this paper it was attempted to explore the best configuration of parameters considering Markov field direction and window size.

Oliveira et al., 2009 proposed a complete workflow for detection and categorization of road surface cracks using imageries from road monitoring vehicles in a fast solution. Morphological operation is used to reduce noise and through dynamic thresholding cracks are generated. Evaluating the results, success rate of 95 percent is achieved.

Sankarasrinivasan et al., 2015 proposed an innovative protocol for full field mapping of a large civil structures involving effective use of Unmanned Aerial Vehicles to enable real time structural health monitoring. The proposed framework integrates UAVs, image processing and acquisition procedures for crack detection and assessment of surface degradation. A novel approach is proposed combining hat transform and HSV thresholding technique for crack detection. In addition, grey scale thresholding is employed for the measurement of surface degradations. The demonstrative model is proven to be reliable and feasible for full field mapping and health monitoring for civil infrastructures.

In summary, it is obvious that remote sensing based methods of crack monitoring usually result in satisfying results in case of time and cost and among them UAV based imageries prompt to be more interesting in recent years. In image processing based methods, pre-processing step is crucial for achieving higher accuracy in final results, otherwise, the results are promising, but they are affected by spots or shadows.

Up to now, a few image based crack detection methods have been studied and proposed specially for UAV based imagery. Most of these studies are focused on cracks in concrete surfaces or simple data sets. In this study we proposed an automatic method for asphalt crack detection in roads which are highly involved with violating objects, noises, spots and so on.

\section{Proposed Crack Detection Method}

Considering the capabilities of information UAV imaging systems provide and on the basis of the researches developed for crack detection in various applications, the proposed method for automatic crack detection is presented. As illustrated in figure 1, the proposed method is comprised of six levels of action. At the first level, aerial imageries along with simultaneous record of onboard GPS/INS measurements of Exterior Orientation parameters and also GCPs are acquired. In the next level, through photogrammetric computation, digital Ortho-Image of the region is generated. Next, in preprocessing section noise reduction, image enhancement and removal of non-crack objects such as vegetation, cars and shades are performed. Through candidate crack detection, a set of hypothesis cracks primitives which enjoy structural similarity to typical cracks are generated using edge detection techniques. In the next level, to verify the true cracks, best features to utilize in classification process are computed and assessed. Then, SVM classification is used to determine final cracks and a 
146

147

148 modification process is proposed. Finally, in the evaluation step, the results are compared with respect to ground truth collected by expert.

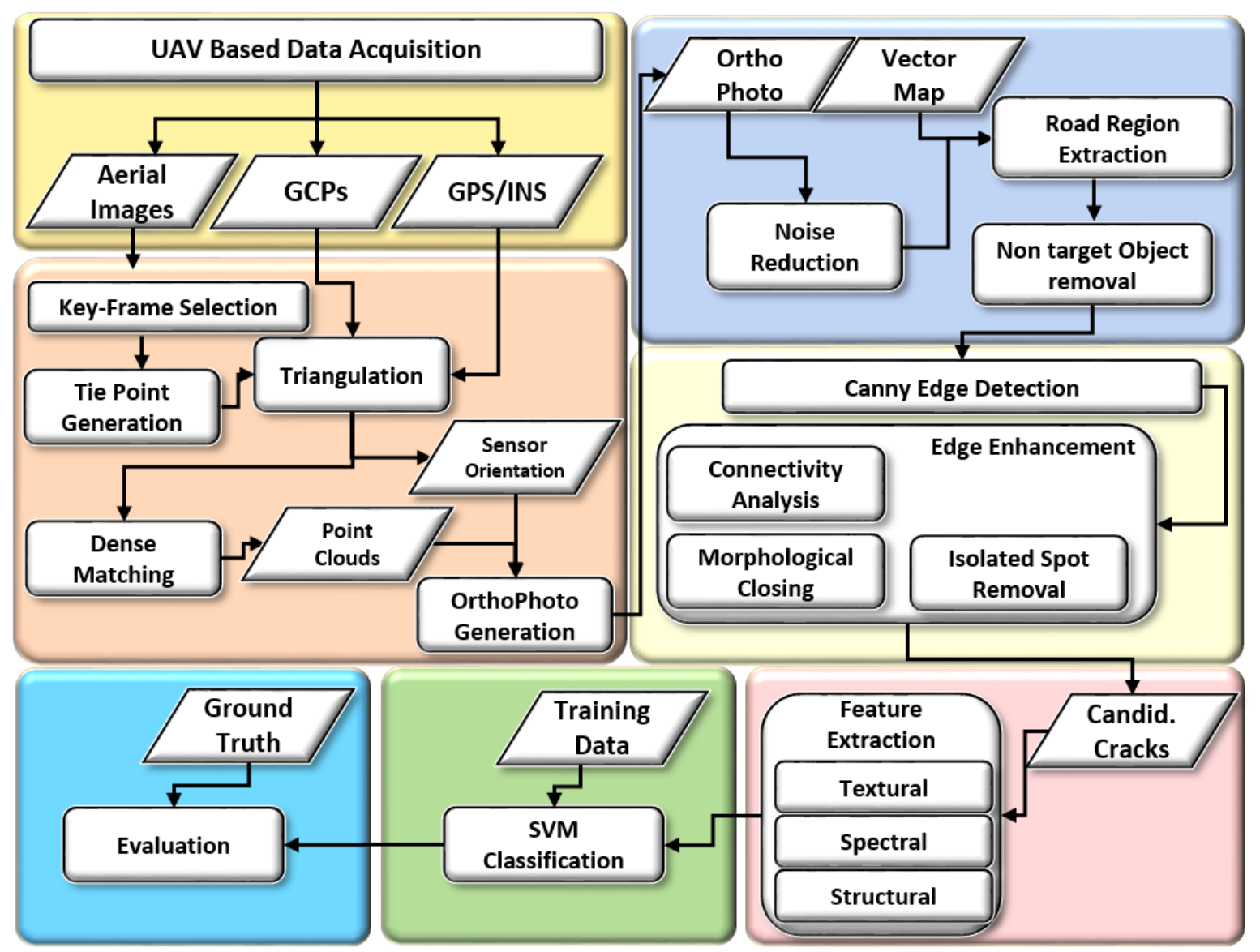

Figure 1. the flowchart of the proposed automatic crack detection method from UAV images

\subsection{UAV Based Imaging and Data acquisition}

As roads are elongated regions, the use of light multirotor and light sensors with high mobility and lower flight altitude and speed are considered. To fortify the process of sensor orientation, onboard GPS/INS information are used. To improve the spatial accuracy of the final map of cracks, GCPs are acquired through ground surveying.

The most important fact about choosing most suitable UAV and sensor is the imaging speed which is related to the sensor speed and UAV altitude and speed. Too low speed increases the project cost and too high speed will result in blurriness of images. On the other hand, the resolution of final achieved images should be considered in advance at the flight planning step. The optimum resolution depends on the width of cracks, if it's less than 3 pixels, the line extraction methods can be applied. Therefore, it should be defined in a way to provide optimum size of crack in images and also prevent data redundancy. The flight time is also important in order to prevent large shades which might result in occluded area.

\subsection{Key-frame Extraction}

As the sensor used is a video acquisition sensor, after data acquisition, key frames have to be extracted. Key frames can be selected based on radiometric and geometric aspects. In radiometric key frame selection, the quality of the frames is checked and the blur low content frames are removed; but in geometric key frame selection, the frames are checked for geometrical stability vital for photogrammetric processing.

In this study, for radiometric frame selection, the BluM metric is applied. The output of this algorithm is a score between 0 to 1 . Results close to 0 represent the best and results close to 1 represent 
172

173

174

175

176

177

178

179

180

181

182

183

184

185

186

187

188

189

190

191

192

193

the worst sharpness of images, respectively. Using a predefined threshold, acceptable and nonacceptable frames are selected (Crete et al., 2007; Arofteh et. al., 2017).

Geometric Robust Information Criterion (GRIC) factor which is presented by Torr et al. (1996) is applied for geometrical evaluation of frames. GRIC evaluates which of the Fundamental epipolar geometry model $(\mathrm{F})$ or homography model $(\mathrm{H})$ is best suited to explain the geometrical relation of two sequences (Arofteh et. Al., 2017). GRIC is defined as:

$$
\text { GRIC }=\sum_{i} \rho\left(e_{i}^{2}\right)+\lambda_{1} d n+\lambda_{2} k
$$

and

$$
\rho\left(e_{i}^{2}\right)=\left\{\begin{array}{cc}
\frac{e^{2}}{\sigma^{2}} & \frac{e^{2}}{\sigma^{2}}<\lambda_{3}(r-d) \\
\lambda_{3}(r-d) & \frac{e^{2}}{\sigma^{2}} \geq \lambda_{3}(r-d)
\end{array}\right.
$$

Where $d=$ Dimension of the selected motion model ( $H$ has the dimension two and $F$ has dimension three); $r=$ Dimension of the data (i.e. four for two views; $k=$ Number of the estimated model parameters (seven for $F$ and eight for $H$ ); $n=$ Number of tracked features; $\sigma=$ Standard deviation of the error on each coordinate; $e_{i}=$ Distance between a feature point transferred through $H$ and the corresponding point in the target image or the Euclidian distance between the epipolar line of a feature point and its corresponding point in the target image.

The GRIC has used for key frame extraction with tuning parameters of $\lambda_{1} \cdot \lambda_{2}$ and $\lambda_{3}$ with:

$$
\lambda_{1}=\ln (r) \cdot \lambda_{2}=\ln (r n) \cdot \lambda_{3}=2
$$

In practice, to avoid selecting too many key-frames, it is proposed to pick a key-frame at the last frame for which:

$$
n i \geq 0.9 n e
$$

where $n i=$ Number of valid tracked features; $n e=$ Number of valid tracks when the Epipolar geometry model overtakes the Homography model.

After initializing the first frame of the sequence as key-frame and proceeding frame by frame, the next key-frame is selected if the GRIC value of the motion model $F$ is less than the GRIC value of $H$.

\subsection{Photogrammetric Computation}

In the photogrammetric computation, the acquired imagery and extracted frames are elaborated to digital Ortho-image. The process as it is so called Structure From Motion (SFM) starts with the extraction of tie points in imagery using image matching usually being performed automatically through point extraction and matching algorithms. Then, block bundle self-calibration is performed, firstly to estimate interior and exterior orientation parameters of images and secondly to estimate 3D ground coordinates of tie points. Then, through dense matching algorithm, Digital Elevation Model of the region is generated. Using a backward projection technique, the Digital Ortho-photo is generated. The flowchart of Photogrammetric Computation step is depicted in figure 2.

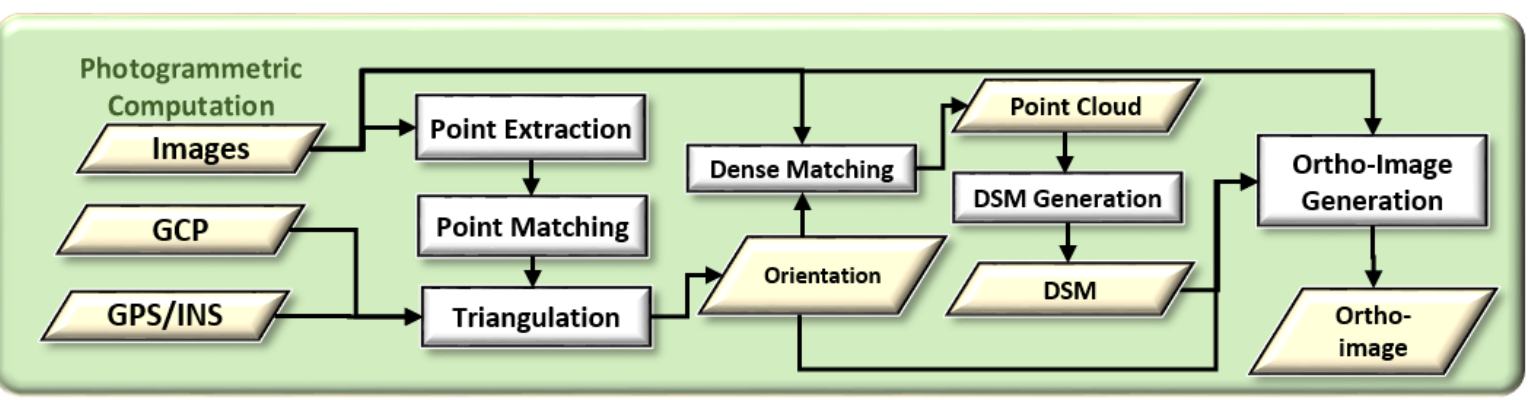


Figure 2. Ortho image generation through Structure form motion procedure.

\subsection{Preprocessing}

The preprocessing section considers two phases of low and high level preprocessing procedures. In the low level process, noise reduction or any further image enhancement and filtering is applied. High level preprocessing includes the removal of non-road regions or regions where the existence of any crack is impossible or improbable or simply not required. In other words, the road region in the digital Ortho-image could be inspected for road regions which is mostly applicable by overlaying digital maps of the road and masking target road regions. On the other hand, there are some nonroad objects on the surface of the road which may violate the process of crack detection. In this case violating non target objects such as cars, shades and signs are removed.

\subsubsection{Image Enhancement and noise removal}

In order to reduce the noisy content of image and to enhance the image in a way that the cracks become more distinctive than the background, this study proposed a combination of smoothing filter and top hat and bottom hat operation based on relation (5).

$$
\mathrm{F}=((\mathrm{SH}+\operatorname{tophat}(\mathrm{SH}))-\operatorname{bothat}(\mathrm{SH}))
$$

where $\mathrm{F}$ is the enhanced image, $\mathrm{SH}$ is the input image which is smoothed based on a Gaussian filter.

\subsubsection{Removing Marginal Non- Road Regions}

In this step, roads regions are extracted from the scene so the process of crack detection is only conducted on the road region to decrease the amount of unnecessary computation and false positive detections. Therefore, road network is projected on the Ortho-image and according to the width of the road segment, the corresponding road regions are extracted. It should be noted that the quality of generated Ortho-image and the accuracy of road network has direct impact on the extracted road region. Therefore, an accurately generated Ortho-image and updated accurate vector road network is necessary.

\subsubsection{Removing Non target disturbing objects}

Road surface presented in the digital Ortho-image is always violated by occlusions imposed by the scene configuration of objects. The existence of vegetation, cars, signs and lines, shades and occlusion from neighboring elevated objects such as buildings, may disturb the process of crack detection through increasing high frequencies to the digital imagery and increasing the number of detected edges and as a results increasing the percentage of False Negative detections.

Threshold applying is the main tool to remove most of the violating objects. For vegetation removal NDVI in case the Ortho-image enjoys Infra-Red channel or Greenness value where only RGB channels are available, are often used (Samadzadegan and Zarrinpanjeh, 2008). For shade removal the same procedure considering illumination in IHS space along with Hue value is used. For car detection, morphological geodesic filtering is believed to be promising [Zarrinpanjeh and Dadrassjavan, 2017]. In this case the size of extracted cars is inspected with respect to conventional car sizes.

\section{5. Crack primitive detection}

The process of crack primitive extraction in this research is mainly based on edge detection techniques. As cracks appearing on the surface of the road pavement demonstrate sudden changes in grey value, it is reflected through high frequencies in the imagery. It should be noted that as high 
frequencies appear in the form of various objects such as dots and ramps, the most important elements in choosing proper detector is extremely vital. According to the linear characteristic of cracks Canny edge detection is used as a solution (Gonzalez and Woods, 2002).

To enhance detected cracks, Connected Component Analysis and Top-Hat morphological filtering are performed. This makes the extracted candidate crack primitives to be enhanced and considered as an image objects rather than pixels. Because the results of edge detection are supposed to be accompanied by similar linear objects such a lines and repaired pavements, crack enhancement helps removing the scattered non-crack edges. Nevertheless, the detected edges also include false cracks that should finally be verified. Smoothing and morphological filters might help removing these artifacts but they might remove sections of cracks as well. As a result, a sliding window filtering method is proposed in this study. Based on the proposed strategy, a window with size smaller than the smallest crack size of the dataset is sliding through the whole image and any objects fully bounded by this window is removed.

\section{6. Crack and non-crack classification}

After extraction of crack primitives those are considered as the candidate cracks are introduced to classification process to define the true cracks and to remove false positives. The classification process is composed of two main stages as feature extraction and SVM classification of extracted features.

\subsubsection{Feature extraction}

To consider SVM classifier to distinguish between crack and non-crack hypothesis primitives, a list of features is considered and computed. In this research, a set of spectral, textural and structural features are studied and used. Table (1) defines the feature to be computed for inspection of extracted candidate cracks.

Table 1. Feature for Crack detection from satellite imagery.

\begin{tabular}{|c|c|c|}
\hline \multicolumn{2}{|r|}{ Feature } & Description \\
\hline \multirow{2}{*}{ Spectral } & $\mathrm{R}, \mathrm{G}, \mathrm{B}$ & Red, Green, Blue Channels of the Digital Othro-image \\
\hline & Mean & Mean value of spectral bands \\
\hline \multirow{4}{*}{ Textural } & Contrast & Contrast of the neighborhood Box of the evaluated pixel \\
\hline & Correlation & Contrast of the neighborhood Box of the evaluated pixel \\
\hline & Energy & Computes the energy of the neighborhood Box [haralick] \\
\hline & Homogeneity & Computes the Homogeneity of the neighborhood Box [haralick] \\
\hline \multirow{5}{*}{ Structural } & Extent & The area of the Bounding Box of the Crack \\
\hline & Eccentricity & The eccentricity value of the Bounding ellipse \\
\hline & Minor axis Length & The Minor axis length of the bounding ellipse \\
\hline & Major axis Length & The Major axis length of the bounding ellipse \\
\hline & Orientation & The angle of the major Axis with respect to horizontal axis \\
\hline
\end{tabular}

Features used in this research are mostly chosen by inspecting the specification and characteristics of cracks. Therefore, more structural features are chosen rather than spectral where the spectral difference of cracks compared to non-crack paved regions is not so prominent.

\section{6.2. SVM Classification}

Training regions are extracted and SVM classifier is used for classification of candidate cracks. Training the SVM classifier, all candidate cracks are introduced to the trained SVM and the extracted 
candidate cracks are evaluated. As SVM is defined to classify patterns in two classes, it is found suitable to detect cracks from non-cracks objects in this application (Cortes and Vapnik, 1995).

\subsection{Evaluation}

To evaluate the successfulness of the results, two levels of evaluation is proposed. In the first one, the capability of SVM classifier in being trained by the training data is evaluated based on the test data set. The test data set is a random selection of pixels as test and training data. Moreover, for accuracy assessment of the result of the proposed automatics crack detection method, they are compared to the cracks detected manually by the expert as independent ground truth.

\section{Results}

To experiment the capabilities of the proposed method in successful detection and mapping of the cracks in the road pavement, the proposed method is implemented and tested for UAV based aerial imagery and the generated results are evaluated and discussed.

\subsection{UAV based Image Acquisition}

For image acquisition, a multirotor UAV equipped with a GoPro4 camera and GPS/IMU systems is used. Table (2) defines the specifications of the platform. Images are acquired from a road in south east of Tehran, Iran. The overall view of selected road and the summary of flight plan parameters are provided in figure (3).

Table2: The aerial platform specification

\begin{tabular}{|c|c|}
\hline Flight Endurance & $40 \mathrm{~min}$ \\
\hline Max Flight Height & $2000 \mathrm{~m}$ \\
\hline Dimension & $1.2 \mathrm{~m}$ \\
\hline Optimum Speed & $20 \mathrm{~km} / \mathrm{h}$ \\
\hline
\end{tabular}
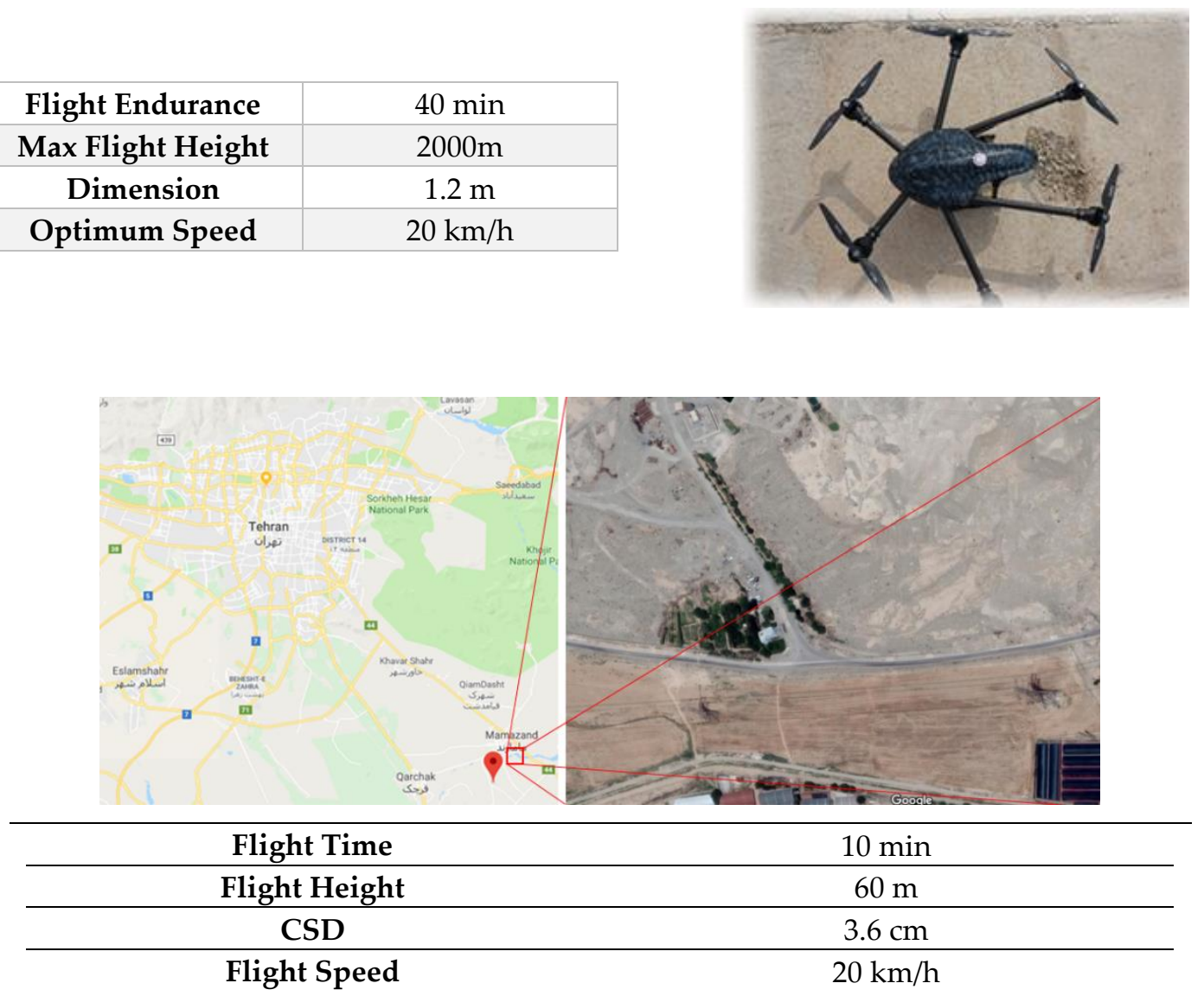
Figure 3. Data set and flight plan parameters

\section{4-2- Key-frame selection}

To execute photogrammetric process key frames are selected from video string and images suffering from lower quality in terms of illuminance, focus and motion blur are removed. Moreover, to prevent data redundancy, key frames are selected based on geometrical stability for bundle block adjustment based on GRIC algorithm. Figure 4, shows some samples of rejected frames.
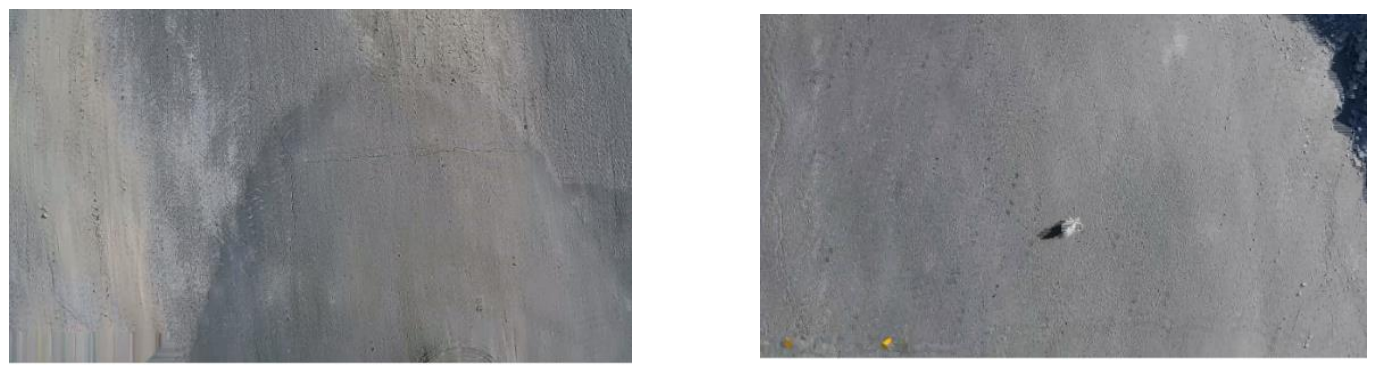

Figure 4. Samples of rejected fames according to BluM procedure.

\section{4-3-Photogrammetric Computation}

As a result of key-frame extraction step, images with minimum 70 percent overlap are selected those are higher in quality and enjoy stable epipolar geometry. Conducting the photogrammetric procedure, the ortho-image of the region is generated. Figure 5, illustrates the camera position and generated ortho-image. The block bundle adjustment is conducted with the accuracy of 0.01 meters.

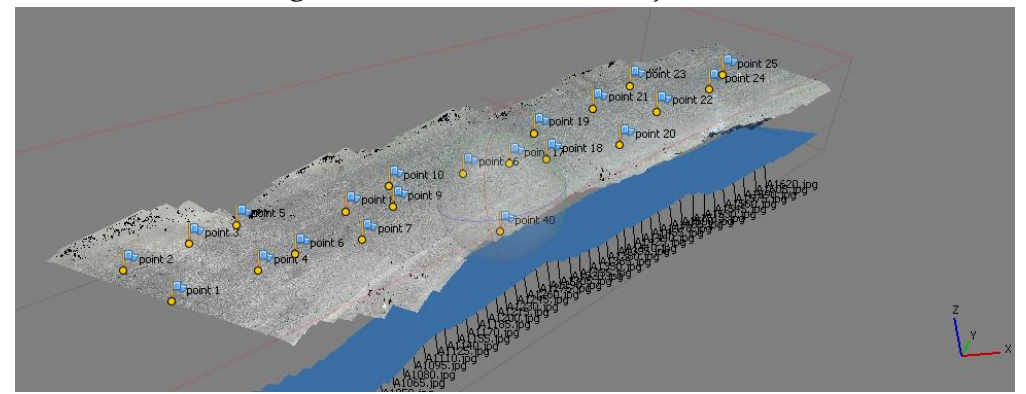

a

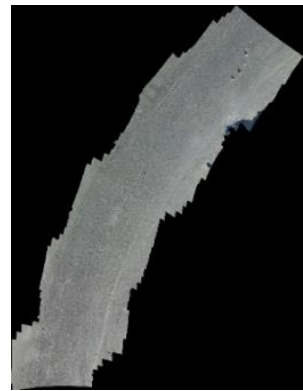

b

Figure 5. a) Photogrammetric process of selected images b) resulted digital Ortho-image.

\section{4-4- Preprocessing}

\section{Image enhancement and noise removal}

In the first step of image preprocessing, image enhancement is applied to reduce noise content of images and also to enhance the image for crack detection based on Canny operation. For the purpose, image is firstly smoothed based on Mean filter and then the combination of Top Hat and Bottom Hat operation is applied as discussed previously. The parameters for these operators are set as $\mathrm{SE}=3$, Sigma $=0.2$ and Hsize $=5$. 


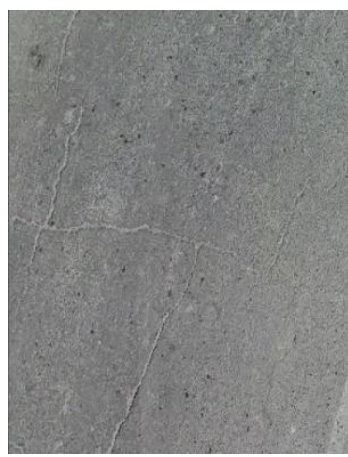

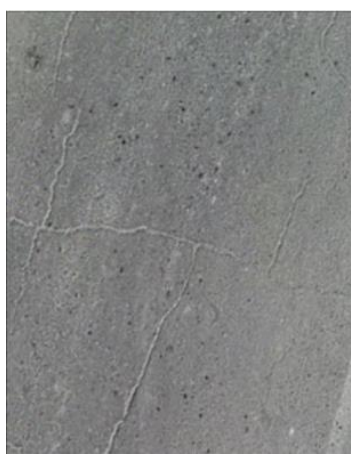

b

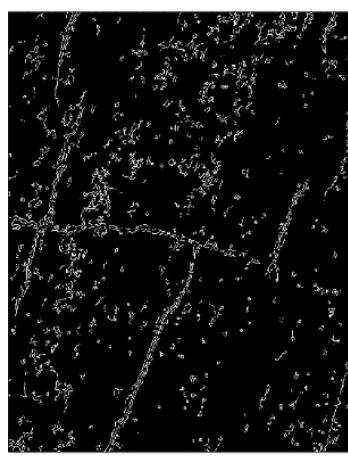

c

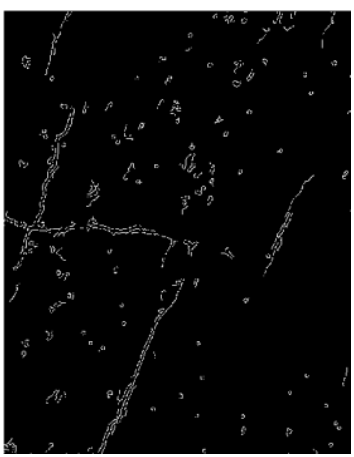

d

Figure 6. a) Original Image; b) Results of Smoothing step; c) Results of Canny edge detection; d) results of canny Algorithm after Top Hat operation

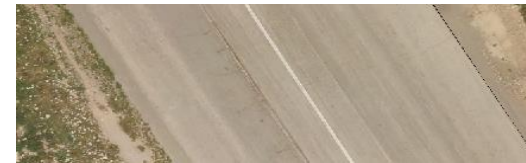

a

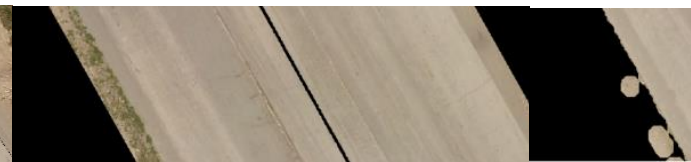

b

\section{Non-target removal}

In this step, non-target regions violating the process of crack detection are omitted. At first, the road segment of image is extracted and the back ground is removed. This process is based on the preinformation about the road map and the width of road based on scale of image. Figure (7) presents the result of road extraction process. The accuracy of this step is evaluated based on the map generated by digitizing the ortho-image by expert which is determined as 97 percent. The main reason of the lower accuracy is the vegetation covering the marginal part of the road which is also removed based on threshold applying on the Red band of images. Doing so, the final quality increased to 98.8 percent. The road white lines can also be removed easily based on applying threshold.

Figure 7. a) Original image; b) road extraction and white line filtering; c)vegetation filtering

The point that might challenge the automatic extraction of cracks is the margin of road after extraction of road from the back ground. This margin should be removed from the image before crack detection process. In figure (8) results of this step is presented. The operation of Canny line extractor before and after this step is also presented to prove the necessity of this process. 

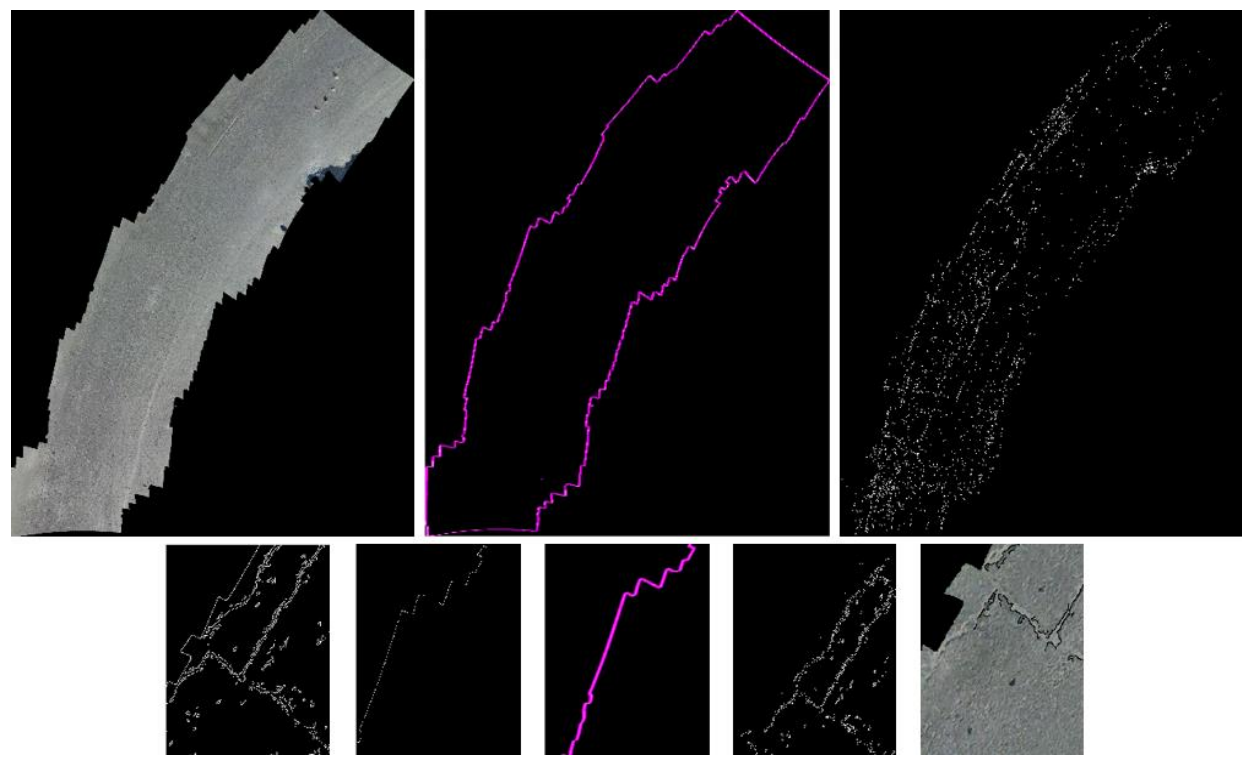

Figure 8. road margin removal

The large shadows on the road are also non objects elements that might violate the crack detection process. For the purpose, shallows are removed based on applying threshold value and remaining isolated small objects are also removed based on morphological filters. Results are presented in figure (9). The successfulness of this process is also evaluated based on expert generated data.
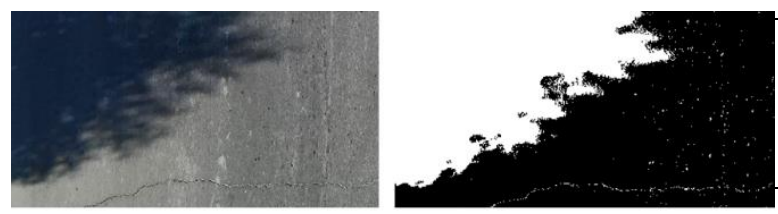

\begin{tabular}{|c|c|c|c|}
\hline \multirow{2}{*}{\multicolumn{2}{|c|}{$\begin{array}{c}\text { Total number of } \\
\text { pixels } \\
1920000\end{array}$}} & \multicolumn{2}{|c|}{ Algorithm result } \\
\hline & & shallow & $\begin{array}{c}\text { Non- } \\
\text { shallow }\end{array}$ \\
\hline \multirow{2}{*}{$\begin{array}{l}\text { Expert } \\
\text { result }\end{array}$} & shallow & 713718 & 22969 \\
\hline & $\begin{array}{c}\text { Non- } \\
\text { shallow }\end{array}$ & 3272 & 1180041 \\
\hline \multicolumn{2}{|c|}{ Total accuracy } & \multicolumn{2}{|c|}{$98.6 \%$} \\
\hline
\end{tabular}

Figure 9. shallow removal and the accuracy assessment

Cars and large non targets elements are also removed by applying geodesic filter following by a

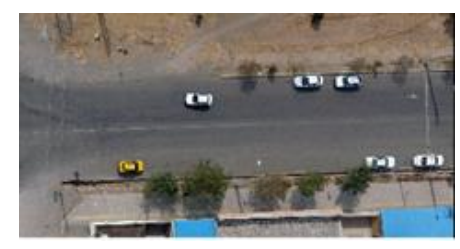

a

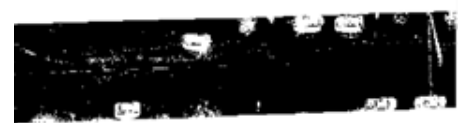

C

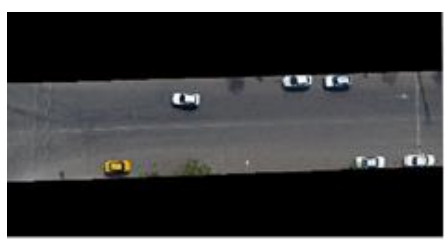

b

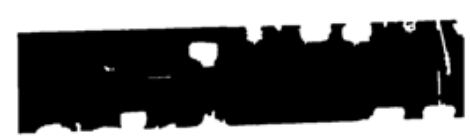

d

\begin{tabular}{|c|c|c|c|}
\hline \multirow{2}{*}{$\begin{array}{c}\text { Total Pixels } \\
36000000\end{array}$} & \multicolumn{2}{c|}{ Algorithm result } \\
\cline { 3 - 4 } & Non-Road & Road \\
\hline \multirow{2}{*}{$\begin{array}{c}\text { Expert } \\
\text { result }\end{array}$} & $\begin{array}{c}\text { Non- } \\
\text { Road }\end{array}$ & 21984175 & 340413 \\
\cline { 3 - 4 } & Road & 456589 & 13218823 \\
\hline \multicolumn{2}{|l|}{ Total accuracy } & \multicolumn{2}{|c|}{$97.7 \%$} \\
\hline
\end{tabular}

Figure 10. Cars and big objects removal and the accuracy assessment 


\section{4-5- Crack primitive extraction}

After extraction of road and removing the non-target elements, Canny edge detection technique is executed and edges are extracted. Through proposed Top-Hat algorithm and the recursive window sliding process, results are enhanced and as it is depicted in figure 11, the results are more similar to cracks through visual inspection, compared to the unfiltered ones, as most of noises which may cause false crack detection are removed. Afterwards, using morphological operation, extracted edges are combined as distinctive objects and are introduced to next processing step as candidate cracks.

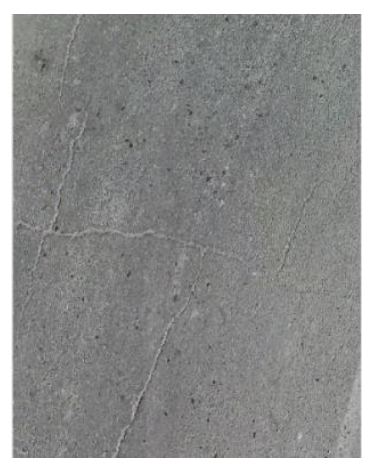

a

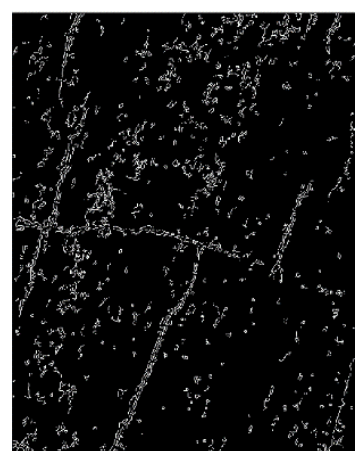

b

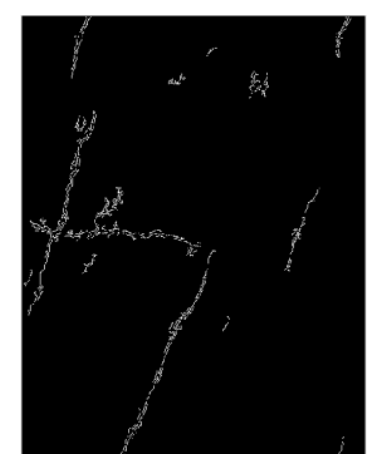

C

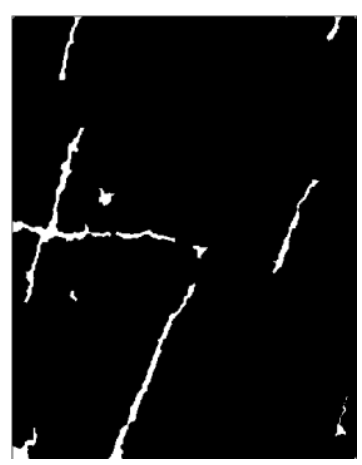

d

Figure 11. a)Original Image; b) Results of Canny edge detection; c) results of canny Algorithm after filtering process; d) final crack candidate elements

\section{4-6-Crack and non-crack classification}

By extraction of crack candidates, the test and training data are prepared. The training data are gathered in two different solution of object based and pixel based as it is depicted in figure (12).
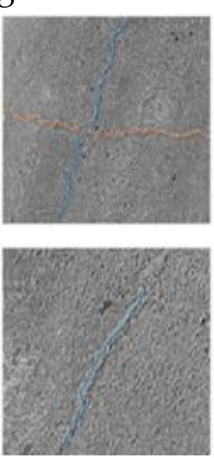

cracks
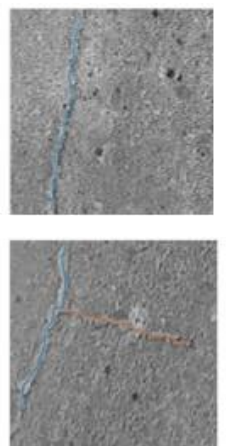

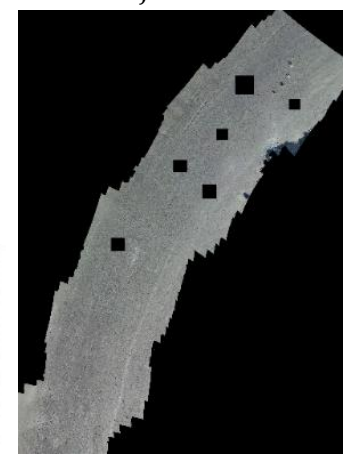

Non-cracks

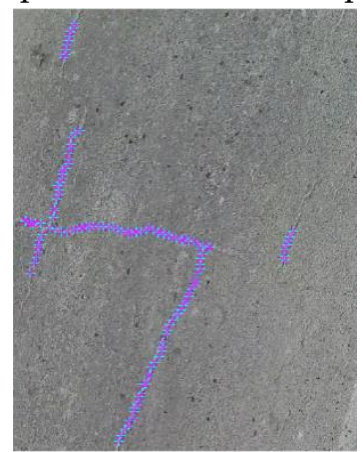

cracks

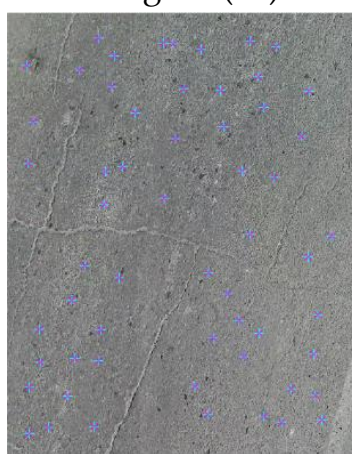

Non-cracks

Figure 12. training data generation, left) object based; right) pixel based strategy

After definition of training and test data sets, candidate cracks are introduced to the SVM classifier. For the classification process, 4 different scenarios of feature selection are implemented and evaluated as Spectral/Textural/Structural and the combination of all features. Achieved results are presented in figure (13). 


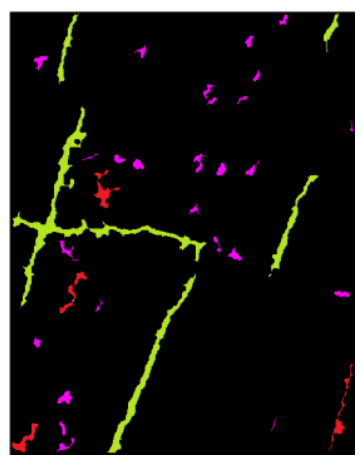

Spectral Features $85 \%$

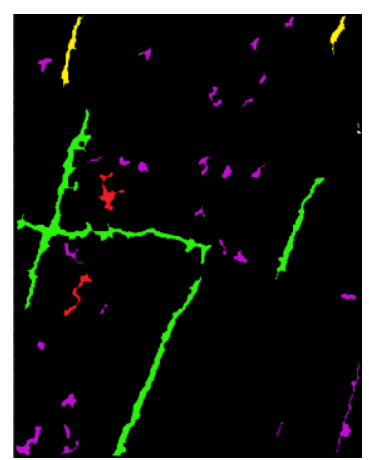

Textural Features $81 \%$

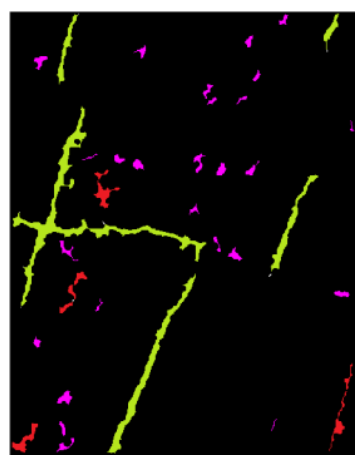

Geometrical Features

$84 \%$

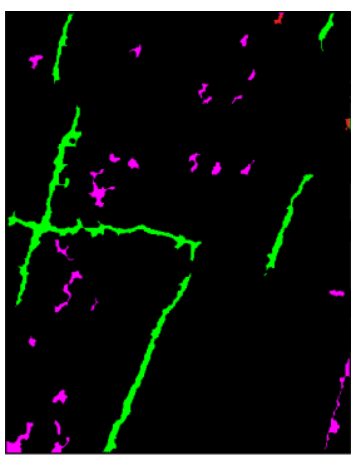

All Features

$92 \%$

Green: cracks; Violet: Non-cracks; Red: Non-cracks labeled as cracks; Yellow: cracks labeled as non-crack

Figure 13. SMV classifier results

In order to improve the results of classification, object level post-processing of results is considered. In this strategy, after classification of the pixels of candidate cracks, the pixel content of each object (candidate crack) is evaluated. By considering the majority and minority condition, the object as a whole is labeled instead of the pixels. Figure (14) depicted the labeling rule in this content. Based on this strategy, the accuracy of test data improved from $87 \%$ to $92 \%$.

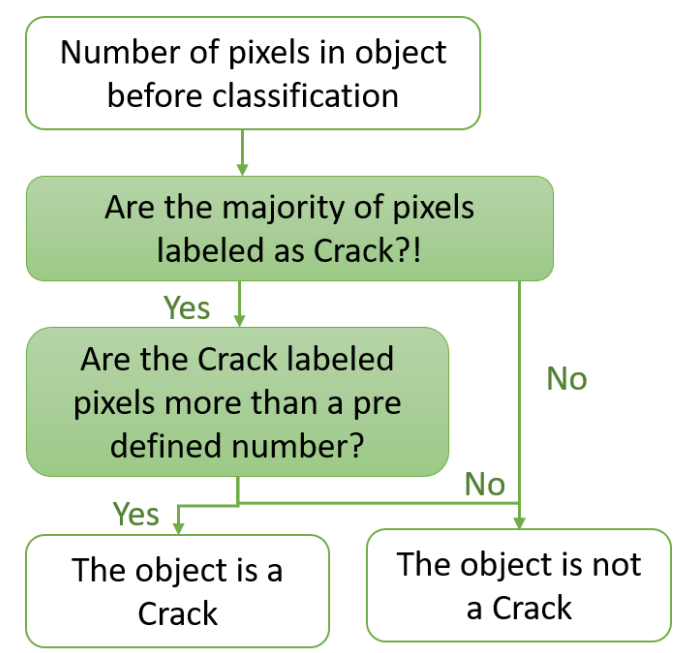

Figure 14. Object based assessment of cracks

\section{4-7-Evaluation of results}

Final results of the proposed crack detection strategy for the whole study area are presented in 


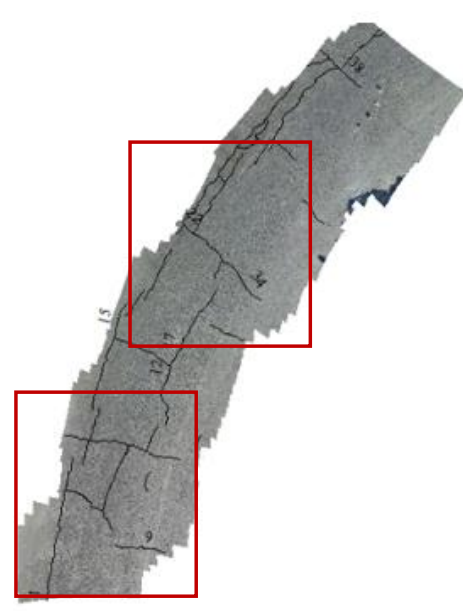

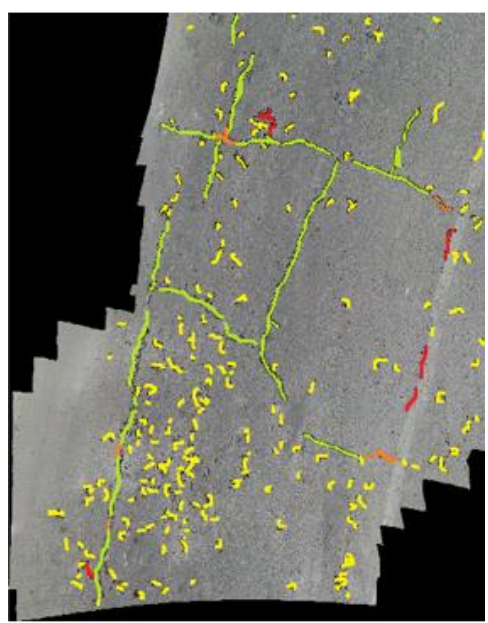

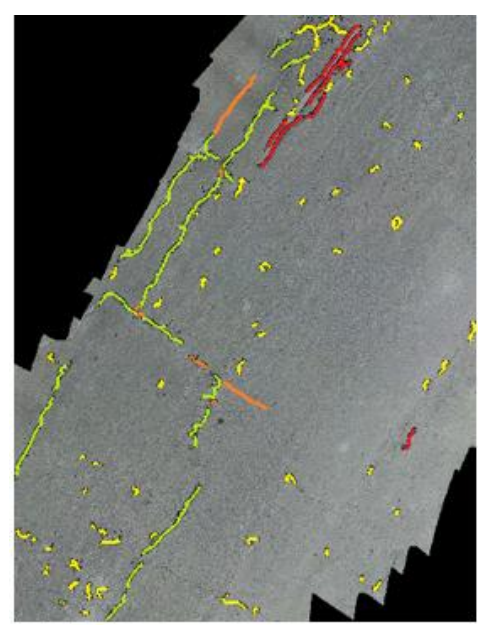

$\mathrm{b}$

Figure 15. Final Crack detection result, a) the ground truth; b) samples of crack detection results, Green: cracks; Yellow: Non-cracks; Red: Non-cracks Labeled as Cracks; Orange: Cracks labeled as Non-crack

Table 3. The validity assessment of the resuls.

\begin{tabular}{|c|c|c|c|}
\hline \multicolumn{2}{|c|}{$\begin{array}{c}\text { Total Pixels } \\
237735\end{array}$} & \multicolumn{2}{c|}{ Algorithm Results } \\
\cline { 2 - 4 } & Crack & Non-Crack \\
\hline \multirow{2}{*}{$\begin{array}{c}\text { Expert } \\
\text { Results }\end{array}$} & Crack & 105012 & 22892 \\
\cline { 2 - 4 } & $\begin{array}{c}\text { Non- } \\
\text { Crack }\end{array}$ & 34955 & 73076 \\
\hline \multicolumn{2}{|c|}{ Total accuracy } & \multicolumn{2}{|c|}{$74 / 9 \%$} \\
\hline
\end{tabular}

\section{Discussion and Conclusion}

Few methods have been proposed to detect cracks from drone images, while there is no certain method which can detect all cracks, automatically. Previous study mostly focused on images taken from concrete structures or by road health monitoring vehicles. These images are high in quality and cracks are usually more prominent than aerial images acquired from roads with asphalt surface.

In automatic crack detection, pre-processing is an important step which plays a critical role in removing violating elements and also decreasing the processing time and cost. Road extraction, image smoothing, noise removal and non-target elements removal are the pre-processing steps discussed in this study.

Moreover, the candidate crack detection is the next step works as pre-crack detection and enhancement. For the enhancement of detected pre-cracks, a recursive strategy based on a sliding window checking extracted objects is proposed which improve the results by removing small non crack objects prominently.

For final classification of candidate cracks in two classes of cracks and non-cracks, SVM classifier with three spectral, textural and geometrical feature classes is applied. Results on test data presented that using textural features leads to the lowest accuracy as $81 \%$ which is predictable because the textural behavior of cracks are not very different from other parts of image specially in asphalt based roads. Moreover, applying spectral and structural features separately resulted in the accuracy about $85 \%$ which is the result of different behavior of cracks in color and shape with respect to non-cracks parts. Finally, applying all the spectral, textural and structural features improve the accuracy on the test data up to $92 \%$.

Moreover, the object level process, which consider the cracks based on Connected Component Analysis as an object, helps to improve the accuracy from $87 \%$ to $92 \%$. 
Finally, the reliability analysis of the proposed strategy was applied based on the ground truth data generated by expert. Results showed the final accuracy of $74.9 \%$. Although this value seems not interesting enough, but considering the complexity of aerial imagery and the non-uniformity texture and variety in shape, size and look of cracks in asphalt roads surface, it could be acceptable for an automatic process. Moreover, results presented that less than 10 percent of cracks are lost, which means that the algorithm missed only a few number of cracks. This failure rate in comparison with the time and cost of the expert based and mobile vehicle based monitoring methods, presents the superiority of the proposed UAV based automatic monitoring of cracks.

\section{References}

Aldea, E., \& Le Hégarat-Mascle, S. (2015). Robust crack detection for unmanned aerial vehicles inspection in an a-contrario decision framework. Journal of Electronic Imaging, 24(6), 061119.

Arofte, a., javidi, n., samadzadegan, f. and dadras javan, f. (2017). geometric comparison of key frame selection techniques in $3 \mathrm{~d}$ reconstruction based on aerial video imagery. SMPR 2017, Tehran.

Behnia, B., Buttlar, W., \& Reis, H. (2018). Evaluation of low-temperature cracking performance of asphalt pavements using acoustic emission: a review. Applied Sciences, 8(2), 306.

Chambon, S., Gourraud, C., Moliard, J. M., \& Nicolle, P. (2010, May). Road crack extraction with adapted filtering and Markov model-based segmentation: introduction and validation. In International joint conference on computer vision theory and applications, visapp (p. sp).

Coenen, T. B., \& Golroo, A. (2017). A review on automated pavement distress detection methods. Cogent Engineering, 4(1), 1374822.

Cortes, C., \& Vapnik, V. (1995). Support-vector networks. Machine learning, 20(3), 273-297.

Crete, F., Dolmiere, T., Ladret, P., \& Nicolas, M. (2007, February). The blur effect: perception and estimation with a new no-reference perceptual blur metric. In Human vision and electronic imaging XII (Vol. 6492, p. 64920I). International Society for Optics and Photonics.

Cubero-Fernandez, A., Rodriguez-Lozano, F. J., Villatoro, R., Olivares, J., \& Palomares, J. M. (2017). Efficient pavement crack detection and classification. EURASIP Journal on Image and Video Processing, 2017(1), 39.

Ersoz, A. B., Pekcan, O., \& Teke, T. (2017, September). Crack identification for rigid pavements using unmanned aerial vehicles. In IOP Conference Series: Materials Science and Engineering (Vol. 236, No. 1, p. 012101). IOP Publishing.

Gavilán, M., Balcones, D., Marcos, O., Llorca, D. F., Sotelo, M. A., Parra, I., ... \& Amírola, A. (2011). Adaptive road crack detection system by pavement classification. Sensors, 11(10), 9628-9657.

Gonzalez, R. C., \& Woods, R. E. (2002). Digital image processing [M]. Publishing house of electronics industry, 141(7).

Grandsaert, P. J. (2015). Integrating pavement crack detection and analysis using autonomous unmanned aerial vehicle imagery (No. AFIT-ENV-MS-15-M-195). Air force institute of technology wright-patterson afb oh graduate school of engineering and management.

Kim, H., Lee, J., Ahn, E., Cho, S., Shin, M., \& Sim, S. H. (2017). Concrete crack identification using a UAV incorporating hybrid image processing. Sensors, 17(9), 2052. 
437 Koch, C., Georgieva, K., Kasireddy, V., Akinci, B., \& Fieguth, P. (2015). A review on computer vision based defect 438 detection and condition assessment of concrete and asphalt civil infrastructure. Advanced Engineering 439 Informatics, 29(2), 196-210.

440 Mohan, A., \& Poobal, S. (2018). Crack detection using image processing: A critical review and 441 analysis. Alexandria Engineering Journal, 57(2), 787-798.

442 Oliveira, H., \& Correia, P. L. (2009, August). Automatic road crack segmentation using entropy and image 443 dynamic thresholding. In 2009 17th European Signal Processing Conference (pp. 622-626). IEEE.

444 Quintana, M., Torres, J., \& Menéndez, J. M. (2015). A simplified computer vision system for road surface 445 inspection and maintenance. IEEE Transactions on Intelligent Transportation Systems, 17(3), 608-619.

446 Samadzadegan, F., \& Zarrinpanjeh, N. (2008). Earthquake destruction assessment of urban roads network using 447 satellite imagery and fuzzy inference systems. The international archives of the photogrammetry, remote 448 sensing and spatial information sciences, 37(PART B8), 409-414.

449 Sankarasrinivasan, S., Balasubramanian, E., Karthik, K., Chandrasekar, U., \& Gupta, R. (2015). Health 450 monitoring of civil structures with integrated UAV and image processing system. Procedia Computer 451 Science, 54, 508-515.

452 Schnebele, E., Tanyu, B. F., Cervone, G., \& Waters, N. (2015). Review of remote sensing methodologies for 453 pavement management and assessment. European Transport Research Review, 7(2), 7.

454 Yin, Z., Mao, Y., \& Seto, C. (2015). Develop a uav platform for automated bridge inspection (No. 25-1121-0003455 295). Mid-America Transportation Center.

456 Yokoyama, S., \& Matsumoto, T. (2017). Development of an automatic detector of cracks in concrete using 457 machine learning. Procedia engineering, 171, 1250-1255.

458 Zarrinpanjeh, N., \& Dadrassjavan, F. (2017). A fuzzy automatic car detection method based on high resolution 459 satellite imagery and geodesic morphology. International Archives of the Photogrammetry, Remote Sensing \& 460 Spatial Information Sciences, 42. 